\title{
Macrocefalias No Quirúrgicas
}

\author{
Dr. Fernando Novoa S. ${ }^{1}$ : Dra. Lidia Troncoso A. ${ }^{2}$; \\ Dra. Marta Colombo C.2; Dr. Fernando Pinto L. 3

\section{Macrocephaly without Endocraneal Hypertension}

\begin{abstract}
A group of 15 patients with macrocephaly without endocrancal hypertension of different etjologics, evolution and prognosis is presented. Macrocephalies are classificd as prinary and those associated to weil defined syndromes or degenerative diseases. They all have an hereditary etiology. Ermphasis is given to the group of Benign megalencephalies. The laboratory techniques to diagnose them including ultra sonography are discussed.
\end{abstract}

El examen del cráneo adquiere gran relevancia en los primeros años de la vida ya que proporciona una información acerca del crecimiento y desarrollo del sistema nervioso central subyacente.

La medición de la circunferencia crateana (CC) es un parámetro clínico esencial y de fácil obtención. Nithaus en $1968^{1-2}$, confeccionó curvas estandarizadas del crecimiento cefálico desde el periodo de recién nacido hasta los 18 años de edad y planteó la necesidad de un seguimiento seriado ${ }^{1-2-3-4}$ para detectar cualquier anormalidad. Sin embargo, es importante enfatizar que basta una medición por sobre 2 desviaciones estândar para obligar al clínico a una evaluación neuropediátrica completa.

1 Jefe Servicio Neuropsiquiatría Infantil, Hospital Paula Jaraquemada.

2 Neurólogo Infantil, Hospital Paula Jaraquemada LNT A.

${ }^{3}$ Neurólogo Infantill, Hospital Paula Jaraquemada.
Macrocefalia es un crecimiento anormalmente acelerado de la cabeza por sobre dos desviaciones estanndar de la curva de Nilhaus ${ }^{1}$, la que puede estar condicionada por: aumento en el contenido (masa cerebral o contenido líquido); aumento en el continente (estructura ósea); combinación de estos factores.

En general, el crecirniento anormalmente acelerado de la cabeza en los primeros años de la vida se relaciona a cuadros de origen quirúrgico (Hidrocefalia, Hematoma Subdural, etc.), pero también puede asociarse a otras patologías no quirúrgicas de evolución no hipertensiva y en la mayoria de las cuales hay un patrón genético hereditario ${ }^{5}$. A este grupo pertenecen las megalencefalias que en general pueden ser: por trastomos en la proliferación, a nivel del segundo mes y cuarto mes de gestación ${ }^{5-6-7}$ por depósito de metabolitos anormales en el cerebro. 5

Para efectuar un estudio adecuado de estas macrocefalias no quirúrgicas se necesita una sos- 
pecha y orientación clínica, medición seriada de la circunferencia cefálica, exámenes de laboratorio de técnica sencilla $y$ no invasiva ( $R x$ cráneo, tamizado metabólico, etc.), la ultrasonografía ${ }^{8-9}$ y la tomografía cerebral sólo si la evolución de la enfermedad lo requiere ${ }^{8 \cdot 9-11}$

El propósito de este trabajo es hacer una revisión de las macrocefalias no quirúrgicas, basados en el análisis de quince pacientes con este problema estudiados en el Servicio de Neuropsiquiatría Infantil del Hospital Paula Jaraquemada.

\section{Clasificación de las Macrocefalias No Quirúrgicas}

Siguicndo la orientación del grupo de Lorber ${ }^{2}$ que se basa en un estudio sobre 557 pacientes con macrocefalia no lipertensiva y con algunas modificaciones en relación a lo publicado (Tabla 1) por otros autores ${ }^{4-5 \cdot 6-12-13}$ se hará la siguiente clasificación:

Megalencefalia Primaria Familiar Benigna, Megalencefalia aislada; Megalencefalia asociada a sindromes conocidos (con talla alta; con talla baja); Macrocefalia asociada a Enfermedades Neuro. degenerativas (Enfermedad de la orina con olor a jarabe de arce, $\mathbf{G M}_{2}$ Gangliosidosis, Enfermedad de Canavan, Mucopolisacaridosis): Hemimegalencefalia. En la Tabla 1 se muestra la clasificación.

\section{Megalencefalia Primaria}

Megalencefalia Familiar Benigna: La macrocefalia está presente desde el nacimiento ${ }^{2 \cdot 3}$ con un marcado predominio en el sexo masculino con respecto al femenino $(4: 1)^{2-3}$, se acompaña de examen neurológico e inteligencia normal 2-3-4-7. La herencia es autosómica dominante con pene- trancia variable $e^{2-3 \cdot 4}$. La incidencia en la población general es de $2 \% .^{2}$

El crecimiento acelerado de ta circunferencia cefálica se produce principalmente en los primeros cuatro meses de vida, siendo en el $80 \%$ de los casos antes de los doce meses de edad. Sin embargo, en un $12 \%$ de los niños esta aceleración puede mantenerse hasta los veinticuatro meses de edad $^{2-4}$. Se describe una mayor incidencia de $\mathrm{Hi}^{\mathrm{i}}$ drocefalia moderada. En estos casos la ultrasonografía como técnica de estudio complementario es de gran utilidad ${ }^{9}$.

En la Figura 1 se muestran los antecedentes clínicos de nuestros pacientes y el árbol genealógico de un paciente en donde sc constata la incidencia familiar de macrocefalia. En estos pacientes la ultrasonografia resultó normal.

Megalencetalia Primaria Esporádica o Aislada: Hay un marcado predominio del sexo masculino (4:1), con herencia autosomica recesiva ${ }^{4-6}$ y un crecimiento acelerado de la cabeza en los primeros años de vida. A diferencia del grupo anterior en un tercio de los casos presentas retardo intelectual, que va desde leve a moderado 2 -3-4-5-6 con alteraciones en el examen neurológico ${ }^{4-5}$. En cinco pacientes con megalencefalia esporádica, controlados desde recién nacido liasta los cinco años de edad, cuatro de sexo masculino y uno de sexo femenino, todos presentaron crecimiento acelerado de la cabeza en los primeros cínco meses de vida. Dos de ellos tenían antecedentes familiares de macrocefalia. De los cinco pacientes, cuatro tuvieron un desarrollo psicomotor y exa. men neurológico normal y el $5^{\circ}$ paciente un desarrollo psicomotor lento y una evaluación neurológica anormal (ataxia, disartria, torpeza moto-

Tabla 1

Clasificación. Macroctâlia No Quirúrgicas.

Primaria Faniliar Benigna

Aislada

1. Megalencefalia

Secundarid
(Asociadas
a sindromes
conocidos)

2. Enfermedades neurodegenerativas

* Enfermedad de la orina con olor a jarabe de arce

* Enfermedad de Canavan

* Gangliosidosis $\mathrm{GM}_{2}$

- Tay Sachs

- Sandhoff

* Mucopolisacaridosis: Enfermedad ae Hurler

* Leucodistrofia metacromática

* Gangliosidosis generalizada $\mathrm{GM}_{1}$
Talta aumentada $=$ gigantismo cerebral
Talla disminuida $=\underset{\text { acondroplasia, }}{\text { rilver }}$
russel!
Síndrome neurocutáneos

3. Tóxicas:

* Pseudo tumor cerebral

* Vitamina A

* Tetraciclina

4. Hemimegalencefatia 
ra fina y gruesa, trastornos de la conducta). Todos tenían separación de suturas en la radiografía de cráneo y la tomografía cerebral practicada en dos de ellos fue normal.

Es importante recalcar que en la etapa adulta, tanto los pacientes con megalencefalia primaria benigna familiar como los casos esporádicos, no necesariamente son macrocefálicos, es más, la mayoría de ellos siguen un crecimiento normal en la curva de Nielhaus ${ }^{1}$ después de los dos affos de edad 4-5.7.

\section{Megalencefalia Sectundaria Asociada a Síndromes Conocidos}

Con talla alta: Síndrome de Sotos o Gigantismo Cerebral: descrito por Sotos en $1964^{14}$.

Son niños de peso de nacimiento por sobre 3.500 grs. con crecimiento somático y ósea excesivo en los tres primeros años de la vida, macrocefalia por sobre dos desviaciones estándar fascies muy característica. Se plantea herencia autosómica dominante de penetración variable, sin embargo hay otros autores que piensan que es autosómica recesiva.
Un hecho constante es que la consanguinidad juega un rol importante $14 \cdot 15 \cdot 16-17$. Se acompaña de retardo mental leve a moderado, con marcados problemas conductuales. Presentan alteraciones de Dermatoglifos, erupción dentaria pre$\mathrm{coz}$ manos y pies grandes. El Electroencefalograma muestra alteraciones inespecíficas, pueden presentar convulsiones ${ }^{17}$.

En estudios de ultrasonografía y/o tomografía cerebral se visualizan ventrículos pequeños, levemente dilatados o normales. Hay mayor incidencia de diabetes en el grupo familiar ${ }^{17-18}$.

Tienen función endoctina normal y su relación ton enfermedad tiroidea es fortuita excepto con enfermedades del tiroides de tipo inmune 19-20. Pueden presentar aminoaciduria alterada ${ }^{17}$. Se asocia a hemihipertrofia que postula de origen cortical o hipotalámico y por esta asociación debe descartarse en niños con sindrome de Sotos la presencia de neoplasias (nefroblastoma, etc. ) ${ }^{17}$. En la Figura 2 se resumen los hechos más relevantes en este sindrome $y$ en nuestro paciente, $y$ la tomografia axial cerebral (TAC).

\begin{tabular}{|c|c|c|c|c|c|c|c|c|c|c|}
\hline & Edad & & $\frac{\mathrm{CC}}{(\mathrm{em})}$ & $\begin{array}{l}\text { Talla } \\
\text { (em) }\end{array}$ & $\begin{array}{c}\text { Antecedentes } \\
\text { Fandares }\end{array}$ & $\begin{array}{l}\text { Desarrutlo } \\
\text { Psicamotor }\end{array}$ & $\begin{array}{c}\text { Examen } \\
\text { Neurológico }\end{array}$ & Radiología & Litrasonografía & $\begin{array}{l}\text { Electroence- } \\
\text { falogralíi }\end{array}$ \\
\hline 1 & $\begin{array}{rr} & \mathrm{R} \\
2 & \mathrm{M} \\
8 & \mathrm{M} \\
15 & \mathrm{M}\end{array}$ & $\begin{array}{l}\mathrm{RN} \\
\mathrm{M} \\
\mathrm{M} \\
\mathrm{M}\end{array}$ & $\begin{array}{l}39 \\
43.5 \\
49 \\
52\end{array}$ & 69 & $(t)$ & Normal & Normal & $\begin{array}{l}\text { Despropor- } \\
\text { ción facial } \\
\text { Separación } \\
\text { suturas } \\
\text { parieto } \\
\text { occipitales }\end{array}$ & $\begin{array}{l}\text { Ventrículos nor- } \\
\text { males } \\
\text { Quiste supraselat }\end{array}$ & Normal \\
\hline 2 & $\begin{array}{ll}15 & M \\
22 & M\end{array}$ & $\begin{array}{l}\mathbf{M} \\
\mathbf{M}\end{array}$ & 49 & $\begin{array}{l}79 \\
50\end{array}$ & $(t)$ & Noimal & Normal & $\begin{array}{l}\text { Despropor- } \\
\text { ción Craneo } \\
\text { facial }\end{array}$ & Nomal & Normal \\
\hline 2 & $\mid \begin{array}{rr}30 & 0 \\
2 & 1\end{array}$ & $\begin{array}{l}\text { Ds } \\
M\end{array}$ & $\begin{array}{l}41.5 \\
42.5\end{array}$ & 57 & $(+)$ & Lento & Normal & $\begin{array}{l}\text { Despropor- } \\
\text { cón Cráneo } \\
\text { facial }\end{array}$ & No Practicada & Normal \\
\hline
\end{tabular}
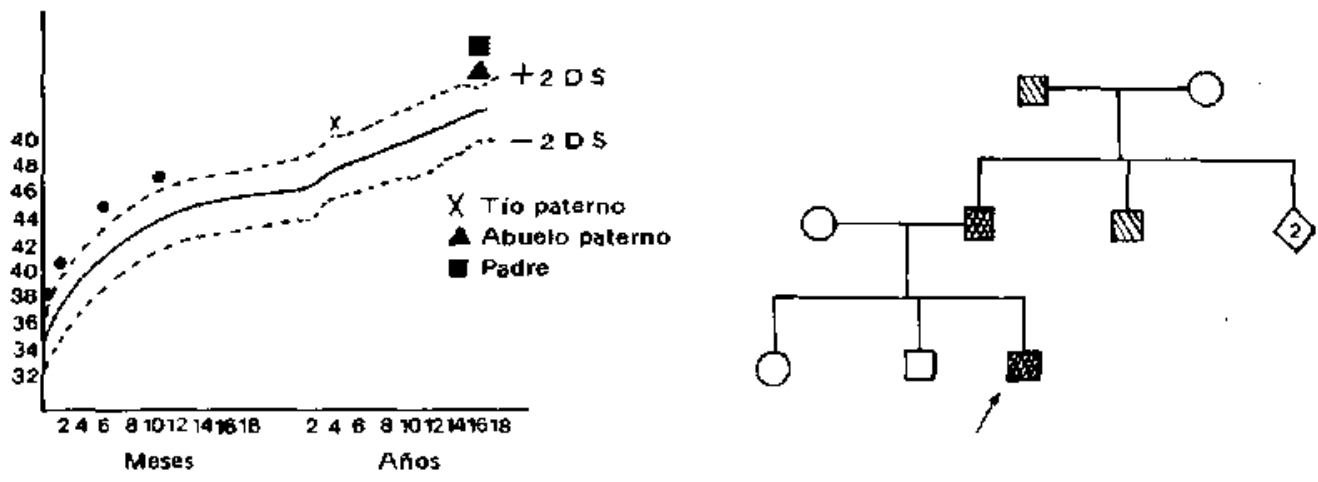

Figura 1.

Megalencefalia Familiar Benigna 


\begin{tabular}{|c|c|c|c|c|c|c|c|c|c|c|}
\hline $\mathscr{\gamma}$ & 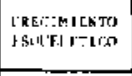 & INTFLICENCIA & $\begin{array}{l}\text { CRANEY } \\
\text { FACIAL }\end{array}$ & DENEN & $\begin{array}{c}\text { OERMAIXXl.1- } \\
\text { FNS }\end{array}$ & $\begin{array}{c}\cos \alpha \\
\operatorname{sic} \cos s\end{array}$ & RAD10LoC IA & $\begin{array}{c}\text { F.LECTRUEY- } \\
\text { CEFALLX.KA- } \\
\text { FIA }\end{array}$ & NTINOSLLLOS & 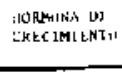 \\
\hline 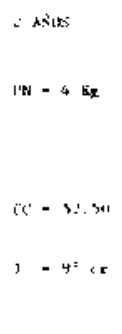 & 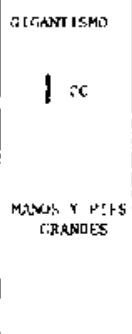 & L:M1TROFE & 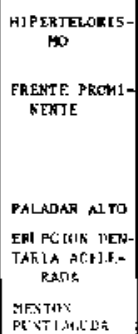 & 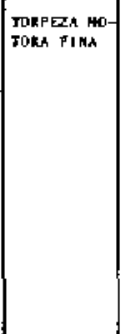 & ALTERRDCS & MCERAL & 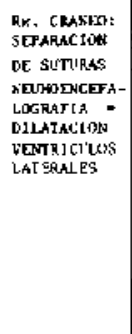 & mokmat & (.) & $s(1+k+4)$ \\
\hline
\end{tabular}
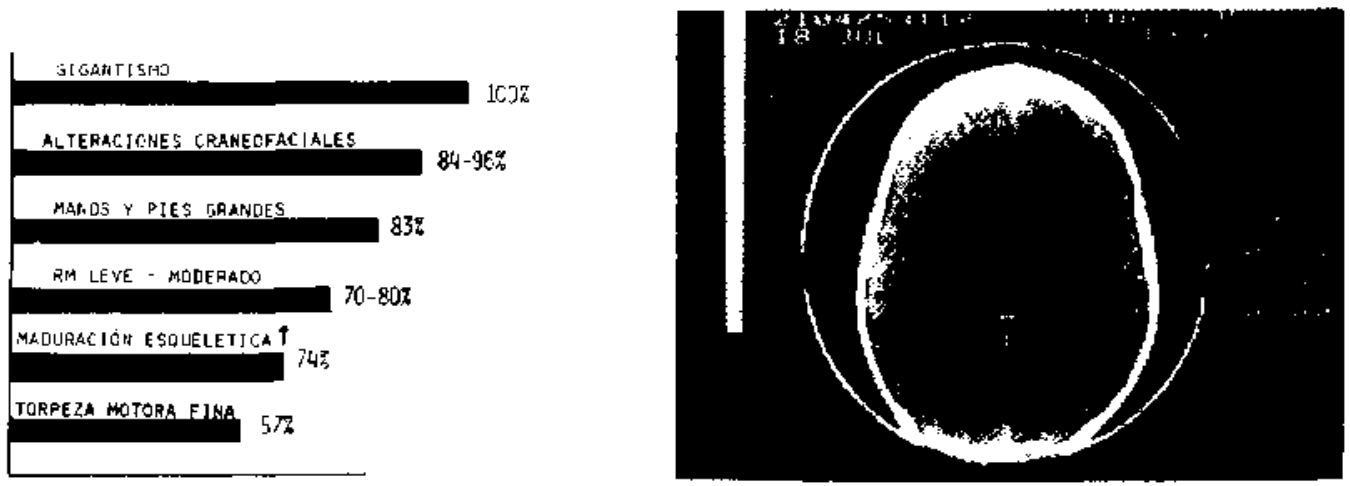

Figura 2.

Sindrome de Sotos

Megalencefalia Secundaria Asociada a Talla Baja .

Acondroplasia: Su herencia es autosómica dominante, pero en $80 \%$ de los casos es una nueva mutación ${ }^{21}$

Es de interés señalar que los antecedentes de padres añosos, infertilidad, mortalidad fetal alta, déficit de concentración de zinc en el cabello, se han relacionado a esta patologia 21 . La macrocefalia de la acondroplasia se produce por altera. ción de la maduración cartilaginosa pero puede asociarse a Hidrocefalia1 1-21-22.

Horton y colaboradores en $1978^{11}$ en un estudio en 400 pacientes con acondroplasia demostró un crecimiento por sobre la curva normal y confeccionó tablas para seguimiento seriado de la circunferencia cefálica ${ }^{21}$. Si el paciente se sale sobre dos desviaciones de estas curvas debe practicarse la ultrasonografía y/o tomografía axial cerebral y plantear la presencia o no de un Hidrocefalia descompensada11-22. En la Tabla 2 se muestra el resumen clínico de un paciente.

Síndrome de Silver Rusell: Se presenta en forma muy esporádica, con igual incidencia en sexo masculino y femenjno ${ }^{23}$. La talla baja (percentil 3 ) es constante; tienen rasgos faciales característi$\cos ^{5-23}$. facie piramidal, comisuras labiales hacia abajo, hipertelorismo, función intelectual nor- mal, excepto un tercio de los casos en que se describe retardo psicomotor moderado a leve, macrocefalia ${ }^{23-24}$. La asimetría está siempre en este sindrome, Cursan con función endocrinológica nomnal ${ }^{23}$ Sólo si se escapan en su crecimiento de talla del tercer percentil es uitil plantear estudios más' especializados, endocrinológi$\cos$ y neuroradiológicos 23-24-25. También se describe marcada precocidad sexual en las niñas con gonadotrofinas aumentadas en suero y ori$\mathrm{na}^{23}$.

En la Tabla 2 se muestran las características clínicas del paciente estudiado y en la TAC se demostró la asimetría en el hemisferio derecho.

\section{Macrocefalia Asociada a Enfermedades Neurode- generativas}

Mucopolisacaridosis: Constituyen un grupo de enfermedades que tienen en común un trastorno en el metabolismo de los mucopolisacáridos. Su herencia es autosómica recesiva. Una de lạs măs conocidas es el Sindrome de Hurler: se caracteriza por un enanismo con facie tosca, hipertelorismo, aplanamiento de la base de la nariz, opacidades corneales, alteraciones óseas y visceromega$\mathrm{lia}^{26}$. Cursan con macrocefalia con mayor diámetro vertical y el cerebro de estos pacientes se en- 
Tabla 2

Megalencefalia Secundaria a Talla Baja.

\begin{tabular}{|c|c|c|c|c|c|c|c|c|c|c|}
\hline $\begin{array}{c}\text { Dingnos- } \\
\text { tico }\end{array}$ & Edad & $\begin{array}{c}\mathrm{CC} \\
(\mathrm{cm})\end{array}$ & $\underset{(\mathrm{cm})}{\text { Talla }}$ & $\begin{array}{l}\text { Desarnollo } \\
\text { Psicomotor }\end{array}$ & $\begin{array}{c}\text { Antectedentes } \\
\text { Familiares }\end{array}$ & $\begin{array}{l}\text { Cránto } \\
\text { Facial }\end{array}$ & $\begin{array}{l}\text { Altejaciones } \\
\text { Ésquciéticas }\end{array}$ & $\begin{array}{l}\text { Dermato- } \\
\text { glifos }\end{array}$ & Radiologia & $\begin{array}{c}\text { Examen } \\
\text { Neurológico }\end{array}$ \\
\hline $\begin{array}{l}\text { Acondro- } \\
\text { plasia }\end{array}$ & $\begin{array}{l}\text { RN } \\
6 \mathrm{M} \\
30 \mathrm{M}\end{array}$ & $\begin{array}{l}54 \\
56\end{array}$ & $\begin{array}{l}43 \\
79\end{array}$ & Lento & $(-)$ & $\begin{array}{l}\text { Djsmor- } \\
\text { fla } \\
\text { Pucnte } \\
\text { nasal an- } \\
\text { cho } \\
\text { Naciz Pla. } \\
\text { na } \\
\text { Paladar } \\
\text { Ojiizal } \\
\text { Ade- } \\
\text { noides }\end{array}$ & $\begin{array}{l}\text { Xifosis Dorso } \\
\text { Lumbar } \\
\text { Pelvis ancha } \\
\text { Genu Varo } \\
\text { Tibjá en sable }\end{array}$ & $(-)$ & $\begin{array}{l}\text { Rx. Cráneo: } \\
\text { scparación } \\
\text { sururas }\end{array}$ & Norrat \\
\hline $\begin{array}{l}\text { Silver } \\
\text { Russell }\end{array}$ & $\begin{array}{l}\mathrm{RN} \\
6 \mathrm{M} \\
30 \mathrm{M}\end{array}$ & $\begin{array}{l}34.5 \\
41.5\end{array}$ & $\begin{array}{l}16 \\
5 ?\end{array}$ & Lento & $(-)$ & $\begin{array}{l}\text { bismat- } \\
\text { fia } \\
\text { Cara: co- } \\
\text { misura la- } \\
\text { bial hacia } \\
\text { abajo } \\
\text { Fontanela } \\
\text { amplia } \\
\text { Asumetría } \\
\text { facial }\end{array}$ & $\begin{array}{l}\text { Desproporción } \\
\text { Cráneo-Talla }\end{array}$ & Alterados & $\begin{array}{l}\text { Edad ósea: } \\
\text { RN } \\
\text { Rx. Cráneo: } \\
\text { Despropar- } \\
\text { ción Oráneo- } \\
\text { Facial } \\
\text { Tac: Leve hi- } \\
\text { pcritofia hemis- } \\
\text { ferio detecho } \\
\text { Ventriculos nor- } \\
\text { males } \\
\text { Ligera aumento } \\
\text { espacio subarac- } \\
\text { noideo }\end{array}$ & $\begin{array}{l}\text { Leve hiper. } \\
\text { tonia } \\
\text { Coef. desatro } \\
\text { lo (Geseb) } \\
3.5 \text { meses }\end{array}$ \\
\hline
\end{tabular}

cuentra aumentado de peso y tamaño. Puede haber engrosamiento de leptomeninges por depósito de mucopolisacárido, lo que da origen a un Hidrocefalia cuadriventricular ${ }^{27-28}$ cuyo pronóstico está en relación al defecto metabólico de base que es de curso progresivo. Deben evolucionarse estos pacientes por su macrocefalia y por la posibilidad de presentar cuadriparesia espástica por compromiso óseo, de columna (xifosis, subluxaciones, etc.). ${ }^{27}$.

En la Figura 3 se muestra la tomografía cerebral y el resumen clinico del paciente y lo descrito en la literatura.

Enfermedad de la orina con olor a Jarabe de Arce: Es un error congénito del metabolismo de los aminoácidos de cadena ramificada por déficit de una decarboxilasa ${ }^{29}$, su herencia es autosómica recesiva ${ }^{29}$. Son niños sanos al nacer hasta los $7-10$ dias de vida en que comienzan a presentar convulsiones, pérdida de los reflejos arcaicos y un olor característico de la orina y cuerpo, semejante al azúcar quemada (Jarabe de Arce). Si no son sometidos a tratamiento dietético adecuado se produce un deterioro progresivo acompañado de macrocefalia por degeneración espongiosa del sistema nervioso central y fallecen en etapas tem. pranas de la vida ${ }^{29 \cdot 30}$. El diagnóstico clínico se comprueba con un estudio de aminoácidos en orina y sangre. Se puede sospechar con el olor característico de la orina y las pruebas de cloruro fërrico y 2-4 difenilhidracina.
Si se plantea el diagnóstico y el tratamiento precoz son niños sanos a futuro.

Enfermedad de Canavan o Degeneración Espongiosa de la Sustancia Blanca: Es una forma de degeneración espongiosa del sistema nervioso central, de probable herencia autosómica recesiva, aunque aún no se sabe con exactitud su pato. genia. Se sospecha que se deba a una alteración en el suministro de energía más que falla metabólica en los lípidos (Tadell y Solitaire, 1972). Son niños normales al nacer pero desde el tercer mes de vida presentan hipotonía generalizada, atrofia óptica, retraso psicomotor, convulsiones (50\%), coreoatetosis y concomitantemente una macrocefalia que se hace más evidente entre el $6^{\circ}$ y $12^{\circ}$ mes de vida. Fallecen alrededor de los dos afios $^{5-31}$. El diagnóstico de la enfermedad de Canavan se confirma por el estudio neuropatológico que demuestra la degeneración espongiosa de la sustancia blanca. Se ha descrito degeneración axonal de nervio periférico.

En nuestro paciente se practicó biopsia cerebral que confirmó el diagnóstico.

Gangliosidosis $\mathrm{GM}_{2}$, Enfermedad de TaySachs y Enfermedad de Sandhoff: Grupo de enfermedades de depósito por trastornos a nivel de enzimas lisosómicas en el metabolismo de lípidos: déficit de hexosaminidasa $\mathrm{A}$ (Enfermedad de Tay-Sachs) y de hexosaminidasa A y B (Enfermedad de Sandhoff). 


\begin{tabular}{|c|c|c|c|c|c|c|c|c|c|}
\hline \multirow{2}{*}{$0_{2.9}$} & \multirow{2}{*}{$\begin{array}{l}\text { lidad } \\
\text { R.V }\end{array}$} & \multirow{2}{*}{\begin{tabular}{|l|}
$\begin{array}{l}C C \\
(\mathrm{Ca})\end{array}$ \\
34 \\
\end{tabular}} & \multirow{2}{*}{$\begin{array}{l}\begin{array}{l}\text { Talla } \\
(\mathrm{cm})\end{array} \\
49.5\end{array}$} & \multirow{2}{*}{ 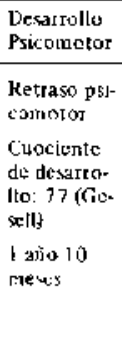 } & \multirow{2}{*}{$\begin{array}{l}\text { Antcidentes } \\
\text { Eamulisés } \\
\text { ( ) }\end{array}$} & \multicolumn{2}{|c|}{ EXAXAtN FISICO } & \multirow{2}{*}{$\begin{array}{l}\text { Radiologia } \\
\text { R.x. Cráneo } \\
\text { Macrocifalia } \\
\text { - Separación de } \\
\text { Suluras } \\
\text { Rx. Colunina } \\
\text { - Vértebtas en Boca } \\
\text { de Pescado } \\
\text { - Xifouis Lumbar } \\
\text { Tat. Dilatación Cur- } \\
\text { driventriculdr }\end{array}$} & \multirow{2}{*}{\begin{tabular}{|c|c|c|}
$\begin{array}{c}\text { Mucopolisasionidos } \\
\text { Onna }\end{array}$ \\
$62.7 \mathrm{mg} / 24 \mathrm{Hrs}$ \\
$\mathrm{N}=20 \mathrm{mg} / 24 \mathrm{Hrs}$
\end{tabular}} \\
\hline & & & & & & 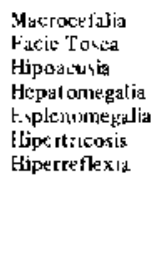 & $\begin{array}{l}\text { Al1ejacjones } \\
\text { Esqueléricas: } \\
\text { Mano en Garrad } \\
\text { Xufose Lumbat }\end{array}$ & & \\
\hline
\end{tabular}

Autosómica Recesiva

Déficit 1-1-Hialuronidasa

Mucopolisacáridos Oruna $(+)$ : Ketaran aumentada - Dermatan aumentado.

\begin{tabular}{|c|c|c|}
\hline & Hidrocefalid & Cardiopatía \\
\hline Peso nacimiento aumentado & & $\begin{array}{l}\text { Talla baja } \\
\text { Déficit Mental }\end{array}$ \\
\hline Macrocefalia - Escafocefalia & Hepa to-Esplenomegalia & Alteraciones Radiológicas \\
\hline Retraso Psicomotor Progresivo Lento & & Opacidad Comeal \\
\hline Anomalidades Faciales Progresivas (Cargola) & & Sordera \\
\hline
\end{tabular}

\section{Macroglosia}

Hirsutísmo

Hernia Unbilical

Xifosis Dotso - Lumbar

$\mathbf{R x}=$ Huesos Distróficos

Cuerpos Vertebrales alterados

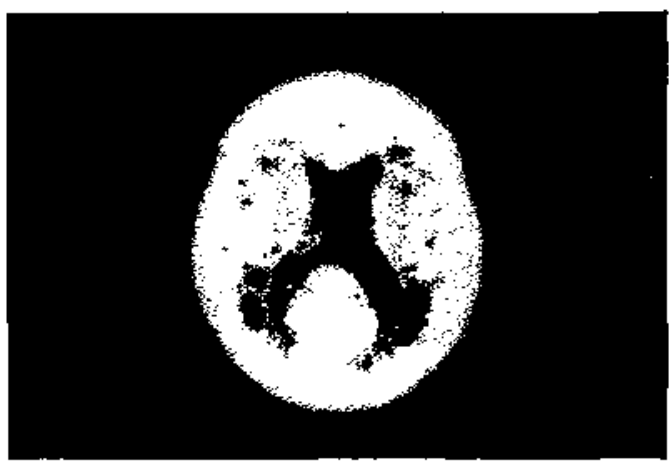

Figura 3

Mucopolisacaridusis. Síndreme de Hurler.

Tienen herencia autosomica recesiva y clínica. mente son niños sanos al nacer y que desde el cuarto al sexto año de vida presentan marcada hiperacusia, deterioro progresivo de su desarrollo psicomotor, amaurosis, con mancha rojo cercza en la región macular. Cursan con macrocefalia que va en aumento y se caracteriza por ser una cabeza grande y pesada. Presentan convulsiones tipo espasmos infantiles y fallecen en ctapas tempranas de la vida ${ }^{32-33-34-35}$. El diagnóstico se confirma por la detección de déficit de hexosaminidasa A o A y B, respectivamente. En este grupo de enfermedades heredodegenerativas se puede hacer diagnóstico prenatal y de heterozigotos. Esta enfermedad tiene marcada incidencia en la raza judía especialmentc Ashke. $n a z i^{5-32-34-35-36}$

\section{Hemimegalencefalia}

Se caracteriza por presentar una asimetría fa- cial y crancana desde el nacimiento, por trastorno en la proliferación celular a nivel del segundo a cuarto mes de vida intrauterina, convulsiones rebeldes a tratamiento y en el electroencefalograma se comprueba hemihipsarritmia. La tomografía cerebral muestra un hemisferio notablemente aumentado y ventrículos asimétricos ${ }^{36}$.

\section{CONCLUSIONES}

En relación a un niño portador de macrocefalia debe plantearse la posibilidad de que se trate de una condición no quirúrgica cuyo reconocimiento puede ser facilitado al considerar los antecedentes familiares $y$ elementos caracteristicos del examen del niño en algunas de estas condiciones.

En su conjunto tienden estas macrocefalias no quirúrgicas a evolucionar sin síntomas de hipertensión endocraneana, siendo su crecimiento más 
acelerado durante el primer año de vida.

En todos estos niños es necesario los controles periódicos desde el punto de vista general del desarrollo y correlacionar la circunferencia cefálica a las curvas de Neilhaus.

En el caso de niños con acondroplasia esta correlación debe hacerse con las curvas de Horton.

En la evaluación inicial tendiente a descartar causas quirurgicas de macrocefalia es de gran utilidad la ultrasonografía de cráneo que tambièn debe ser usada en controles seriados.

Entre las causas más frecuentes de macrocefalias no quirúrgicas está la Megalencefalia Familiar Benigna que se presenta en mayor proporción en el sexo masculino y evoluciona con indemnidad en esfera intelectual.

En el grupo de macrocefalias no quirúrgicas asociadas a enfermedades neurodegenerativas es importante un diagnóstico oportuno por la posibilidad de dar un consejo genéticu c incluso tratamiento que modifique el curso de la entermedad como es en el caso de la orina de olor a jarabe de Arce.

\section{RESUMEN}

Se presenta un grupo de 15 pacientes con macrocefalia de evolución no hipertensiva pero de diferentes ctiologias, evolución y pronóstico.

Se analizan las macrocefalias de origen primario y las asociadas a sindromes conocidos y Et1fermedades Degenerativas del sistema nervioso central. Todas tienen un rasgo genético hereditario.

Se lace énfasis en el grupo de megalencefalias benignas.

Las técnicas de laboratorio para diagnosticarlas $y$ hacer seguimiento de la macrocefalia especialmente utilizando la ultrasonografia como método no invasivo y de alta eficiencia.

\section{REFERENCIAS}

1 Neithatu, G.: llead circumference from birth to eighteen years. Pediatrics 41: 106, 1968.

2 Lorber, J. Priesfley, B: Children with lares leads. A practical approach to diagnosis in 557 children, with special reterence to 109 children with Megslencephaly, Divelop. Med. Child. Neurol. 23: 494. 1981

${ }^{3}$ Etlison, P.: Re uvaluation of the approach to an enlarging licad in infancy. Develop. Med. Child Neurol., 20:738, 1978

4 Asch. A, MD, Myers, G. MD.: Beninen familial macrocephaly: Report of a family and revicw of the litcrature. Pediatries $57: 535,1976$.

5 Rubinstein, J. MD. Warkary, J. MD.: Congenital malformations: Megalencephaly. En: Mental Retardation, New York Mac Millan $128 \quad 155,1975$.
6 Volpe, f.: Neurology of the newborn. Major problems in Clinical Pediatrics, vol. XXII, 3I-34, 1981 .

7 Schreier, H. MD. Rapin. I. MD and cols.: Familial megalencephaly of Ihydrocephalus. Neurology 24: 232. 1974 .

B Kinsiey, D., Kendall, B.: The value of computed tomography in the evaluation of the enlarged head. Neuroradiology 15: 59, 1978.

9 Morgan, C. Ph. D, MD. Trought, W. MD and cols: Comparison of Gray--Scale Ultrasonography and computed tomography in the evaluation of Macrocrania in infants. Radiology $32: 119,1979$.

10 Donat, f. MD.: Eqaluation of macrocrania using computed tomography. An. J. Dis. Child. 135: 1118,1981 .

1 I Hotton, W., Rater, J. and cols: Standard growth curves for achondroplasia. J. Pediatr. 93: 435, 1978.

12 Swaimen. $K$. MD, Wright, F, MD.: The practice of pediatric neurology, Editado por: Samuel E. Harsliterger, Mosby Str. Louis Missuri, U.S.A. $429-435,1982$

13 Weaver, D. MD. Christian, J, Ph.D. HD.: Fumilial variation of head size and adjustment for parenteral head circumfirence. J. Pediatr. 96: 990, 1980.

I Sotos, J.F., Dodge and cols.: Cerebral gigantion in childliond. N. Kngl. 1. Med. 271: 109, 1964.

15 Sotos, J. and Cutler, E.: Cersbral giguntism. Ain. J. Dis. Child. 131:625, 19?7.

16 Sterenson, D. WD. Hopper, A, MD and cols.: Macrosomia: Causes and consepuences. J. Pediatr ] $00: 515,1982$.

${ }^{17}$ El Shafie, M. MO, and Klippel, Ch, Jr. MD.: Sotar Syndromc. En: Associated Congenital Anomalies. Williams/Wilkin, Baltimorc, London, 90-.96, 1481

3B Freeman, J.: Cercbral gipantism, En: Birdt Dufects Compendium. Editado por Danicl Bergsma, Boston Masqacluesetis, 137, 1979.

${ }^{19}$ Wilsom, T. MD, Neufeld, M. MD. Cusebral gigantism and thotoxicosis. J. Pediatr. 96: 685, 1980

20 Frasier, $D$. inD.: Growth disorders in elildren. Pediat. Clín. N. Aner. 26: 12, 1979.

21 collips, $P$. . Chen, $S$, and cols.: Zinc deficiency in achondroplastic cluldren and their parents. J. Pediatr. 94: 609. 1979.

22 Langer, L., Rimoin. D. Acliondro-dasia. En Birth Defects Compendum. Daniel Bergsma, Boston Massaclusctss, 10 1 I, 1979

23 Silver. Henty.: Silver Syndrome. lin: Birth Defects Compondium. Editado por Daniel Bergsman, Boston. Massachusetts, 958-959, 1979.

${ }^{24}$ O'Brien, B., Sadeghi, A. and cots.: Growth bormono deliciency in a patient with Silver Rusell sy nodrome. J. Pediatr. 93: 152, 1978.

25 Drazrim, M. MD. Stclling, M. MD, and cols, SilverRusell syndrome and eraniopharingioma. J. Pediatr. 96: 887,1980 .

26 Steversom. R.: Mucropolysuccharidosis I H. En: Birtil Defects Compendium. Editado por Danitl Bergsman, Bostots. Maswachusetts, 674 675, 1979.

27 Brill, Ch, Rose, J. and cols. Spastic quadriparesis due to $\mathrm{C}_{\mathbf{l}} \cdot \mathrm{C}_{2}$ subluxation in Hurker Syndrome. $\mathrm{J}$. Pediatr. 92: 441.1978.

28 Shimar, S. Singer, H. and cols,: Acute hydroctphalus in Hurler's Syndrome. Ant. J. Dis. Child. 136 556.1982.

29 Scriver. Ch., Rosenberg, t.: Amino acid metibolism and its disorders. Major problems in elinical pediatrics IX, 267--301, 1973. 
30 Volpe: Neurology of the newborn. Major problems in clinical pediatrics. XXII 357-361, 1981 .

31 Allen Richard. Canavan disease. En: The practice of pediatrics neurology. Editado por Samuel E. Haishlerger, Mosby St. Loujs Missouri, U.S.A. $893.894,1982$.

32 Swaisman $K$.: Lipid diseases of the central nervous systcm. En: The practice of pediatrics neurology. Editado por Samuel E. Harshberger, Mosby, St. Louis Missouri, U.S.A. 515-522, 1982.

33 Dolodny Edwin, MD.: Ly sosomal storage disease. N.
Engl. J. Med. 294: 121?, 1976.

34 Dykern, P. MD. Krowjecki, N. MD.: Neurodegentrative diseases of infancy and childhood. Ann. Neurol. 13: 351, 1983.

35 Von Specht B.V., Geiger, B. and cols.: Enzyme replacement in Tay-Sachs disease. Neurology 29: $848,1979$.

36 Tiam, A., Schenk, V. and cols.: Infantile spasm associated with hemihypsarhytmia and hemimegalencephaly. Develop. Med. Cuild. Neurol. 20: 779 , 1978. 